\title{
Deaths linked to synthetic cannabinoids
}

\author{
Dimitri Gerostamoulos $^{1} \cdot$ Olaf H. Drummer $^{1} \cdot$ Noel W. Woodford ${ }^{1}$
}

Accepted: 9 February 2015/Published online: 15 March 2015

(C) Springer Science+Business Media New York 2015

Over the last 5 years there has been an influx of novel designer drugs (mostly illegal) which are intended to mimic the effects of cannabis (synthetic cannabinoids). Many of these compounds are created by research groups attempting to find an analog of cannabis that can be used therapeutically to treat pain and other conditions.

PB-22 (1-pentyl-8-quinolinyl ester-1H-indole-3-carboxylic acid) is a relatively new synthetic cannabinoid which has cannabis like activity and possibly other as yet unknown effects. There is no published data on the pharmacodynamics, pharmacokinetics or toxicology of PB-22. However, a recent publication has reported 4 deaths associated with use of 5F-PB-22 which is a derivative of PB-22 [1]. Although synthetic cannabinoids have been reported in association with sudden death, the precise pathophysiological mechanisms by which death occurs remain obscure.

We have identified three cases in Victoria over a recent 4 month period all of which have PB-22 as the main toxicological finding. In all three cases the cause of death was unascertained following the performance of a full autopsy with ancillary investigations. The subsequent identification of this substance raises the question of its possible involvement in the deaths, particularly in the absence of a competing cause of death.
We remain unsure about the contribution of PB-22 to these deaths however all 3 deaths (ages 15-35) occurred at home in the setting of using this drug with no other competing cause of death identified. We feel it important that practitioners be aware so they can alert patients who may be experimenting with or using designer drugs such as synthetic cannabinoids. The safety of these substances is largely unknown; and those with an existing heart condition may be at risk of sudden death when using drugs which can produce unwanted increases in heart rate and blood pressure.

We thank the State Coroner of Victoria, Judge Ian Gray for allowing this issue to be publicized, and the staff pathologists of the Victorian Institute of Forensic Medicine.

\section{Reference}

1. Behonick G, Shanks KG, Firchau DJ, Mathur G, Lynch CF, Nashelsky M, et al. Four postmortem case reports with quantitative detection of the synthetic cannabinoid, 5F-PB-22. J Anal Toxicol. 2014;38(8):559-62.
Dimitri Gerostamoulos

dimitri.gerostamoulos@vifm.org

1 Department of Forensic Medicine, Monash University and Victorian Institute of Forensic Medicine, Melbourne, Australia 